\title{
Does providing hereditary breast cancer risk assessment support to practicing physicians decrease the likelihood of them discussing such risk with their patients?
}

Awareness, concern, and desire for feedback about heritable risk is high among women having a family history of breast cancer ${ }^{1}$ and feedback in primary care is appreciated by such women ${ }^{2}$ We developed a brief tool for physicians to rapidly assess breast cancer family history with the hopes of providing the means for physicians to provide feedback, generally reassuring, to their female patients having a family history of breast cancer.

The intervention consisted of a coat pocket laminated card including the following: (1) brief risk stratification criteria adopted by all of our local genetic counselors for identification of family histories conferring either high risk of breast cancer or high risk of carrying a BRCA mutation, (2) lifetime probability benchmark ranges (general population, 1st degree relatives of individuals diagnosed with breast cancer, and BRCA1 or BRCA 2 carriers), (3) USPSTF screening recommendations, and (4) local genetic services contact numbers for both formal and informal ("curb-side") consultation. Physicians also received the AMA Monograph, Managing Inherited Breast Cancer Risk.

We conducted a small feasibility study in late 2003/early 2004 among 15 primary care physicians (general internal medicine or family practice) from separate practice locations who had previously identified themselves as being interested in participating in practice-based research. Seven of these $15 \mathrm{com}$ pleted a consent form, a baseline questionnaire, and a 3-month follow-up questionnaire. Our results showed that 5 out of the 7 completing physicians reported a decrease in the frequency of discussing inherited risk with their patients having a family history of breast cancer and the remaining 2/7 reported no change in frequency. We also assessed the impact of the intervention on the physician subjective threshold for classifying a woman as "high risk." Six out of the seven reported an increase in this subjective threshold.
This study was intended only as a feasibility study. We did not sample nor follow-up in a manner to effectively test the efficacy of the intervention. However, these results still give us pause. It appears that we might have had the unintended impact of inserting a barrier to physician-patient discussions about familial breast cancer risk. One plausible reason is that we provided the physician with enough information for them to determine that the majority of the family histories that they encounter are unlikely to confer significantly increased risk to their patients and therefore not worth taking the valuable visit time to discuss it. Given the growing research describing the desire that women have for more feedback about such familial risk in primary care practice and the potential teachable moment that assessing family history provides for cancer prevention, interventions that create a barrier to feedback should be discouraged. We conclude that the intervention we have pilot tested has the potential to cause harm in its current state and needs major revision and reconsideration before conducting a clinical trial. We hope that other researchers who are developing such interventions to support primary care physicians with addressing genetic risk in practice can benefit from our findings.

Robert Gramling, MD Christine Duffy MD, MPH Sean David MD, SM

Brown Medical School Providence, Rhode Island

\section{References}

1. Chalmers K, Marles S, Tataryn D, Scott-Findlay S, Serfas K. Reports of information and support needs of daughters and sisters of women with breast cancer. Eur J Cancer Care (Engl) 2003;12:81-90.

2. Warner E, Carroll JC, Heisey RE et al. Educating women about breast cancer: An intervention for women with a family history of breast cancer. Can Fam Physician 2003;49: $56-63$. 